\title{
Compensation effects of coated cysteamine on meat quality, amino acid composition, fatty acid composition, mineral content in dorsal muscle and serum biochemical indices in finishing pigs offered reduced trace minerals diet
}

\author{
Miaomiao Bai ${ }^{1,2}$, Hongnan Liu ${ }^{1,3^{*}}$, Kang $\mathrm{Xu}^{1,3}$, Xiaofeng Zhang ${ }^{4}$, Baichuan Deng ${ }^{2}$, \\ Chengquan Tan ${ }^{2}$, Jinping Deng ${ }^{2 *}$, Pingping Bing ${ }^{5} \&$ Yulong Yin ${ }^{1,2,3^{*}}$ \\ ${ }^{1}$ National Engineering Laboratory for Pollution Control and Waste Utilization in Livestock and Poultry Production, Hunan Province Key \\ Laboratory of Animal Nutritional Physiology and Metabolic Process, Hunan Provincial Engineering Research Center of Healthy Livestock, \\ Key Laboratory of Agro-ecological Processes in Subtropical Region, Institute of Subtropical Agriculture, Chinese Academy of Sciences, \\ Changsha 410125, China; \\ ${ }^{2}$ College of Animal Science, South China Agricultural University, Guangzhou 510642, China; \\ ${ }^{3}$ Hunan Co-Innovation Center of Animal Production Safety, Changsha 410128, China; \\ ${ }^{4}$ Hangzhou King Techina Technology Company Academician Expert Workstation, Hangzhou King Techina Technology Co., Ltd., \\ Hangzhou 311107, China; \\ ${ }^{5}$ Academics Working Station, Changsha Medical University, Changsha 410219, China
}

Received May 6, 2019; accepted May 21, 2019; published online August 15, 2019

Citation: Bai, M., Liu, H., Xu, K., Zhang, X., Deng, B., Tan, C., Deng, J., Bing, P., and Yin, Y. (2019). Compensation effects of coated cysteamine on meat quality, amino acid composition, fatty acid composition, mineral content in dorsal muscle and serum biochemical indices in finishing pigs offered reduced trace minerals diet. Sci China Life Sci 62, 1550-1553. https://doi.org/10.1007/s11427-018-9399-4

Dear Editor,

Trace mineral elements (ME) and amino acids have been demonstrated to be essential bioelements in animal nutrition. If there is a deficiency in $\mathrm{ME}$, a number of biological functions in animals may be affected, including physical growth, psychomotor development, and immunity. Supplementation of pig diets with $\mathrm{ME}$ is known to improve the animals' growth, reproduction, and their immunity against oxidative stress and cell damage. Pigs that are fed diets with high-dose supplemental $\mathrm{Zn}$ or $\mathrm{Cu}$ have been shown to have an increased $\mathrm{ADG}$ and a decreased F:G ratio (Zhu et al., 2011). However, the pigs' ability to digest and absorb trace elements is limited, which causes environmental con-

*Corresponding authors (Yulong Yin, email: yinyulong@isa.ac.cn; Jinping Deng, email: dengjinping@scau.edu.cn; Hongnan Liu, email: liuhn@isa.ac.cn) tamination and restricts the development of animal husbandry. Therefore, formulation of diets with reduced trace mineral $(\mathrm{Cu}, \mathrm{Fe}, \mathrm{Mn}$, and $\mathrm{Zn}$ ) supplementation may address this problem without affecting animal performance during the finishing period. However, a deficiency of $\mathrm{Zn}$ can lead to failure in the synthesis of RNA, DNA, and proteins. Copper is a crucial component of myoglobin, haemoglobin, and the cytochromes, and is needed in the utilization of iron for protein synthesis. In addition, manganese has been reported to elevate the expression and protein levels of manganese superoxide dismutase ( $\mathrm{Li}$ et al., 2016). It has also been reported that deletion of minerals during the last 6 weeks before slaughter has negative effects on pork quality (Ma et al., 2016). However, the effects of deletion of ME on protein and mineral deposition have not been investigated extensively.

Cysteamine, a new type of feed additive, influences protein digestion and absorption by regulating amino acid 
transporters. It is usually used as a feed supplement in animal production in the form of coated cysteamine (CC). Cysteamine has been shown to play two important roles, namely regulation of mTOR signalling for modifying protein metabolism and protection of cells from oxidative stress (Bai et al., 2018; Zhou et al., 2017) (Figure 1). Previous studies have shown that antioxidants can boost the utilization of iron (Lönnerdal, 2000) and demonstrated the role of cysteamine in delaying meat discoloration through enhanced antioxidative activity. However, the effects of CC on utilization of trace minerals in muscle have not been elucidated. The objective of the present work was to investigate the effects of reduced dietary $\mathrm{ME}$ on meat quality, amino acid composition, fatty acid composition, mineral content in dorsal muscle, and serum biochemical indices in finishing pigs, as well as to test the ability of supplemental $\mathrm{CC}$ to compensate for these effects in finishing pigs. We hypothesized that reduction of $\mathrm{ME}$ or addition of $\mathrm{CC}$ has the potential to affect mineral retention and amino acid metabolism, and that $\mathrm{CC}$ may have a compensatory effect in finishing pigs that have been fed a reduced trace minerals diet.

We designed the feed experiment that 360 crossbred (Duroc $\times$ Landrace $\times$ Yorkshire) finishing gilts and barrows (180 gilts and 180 barrows) with an initial body weight (BW) of $103.9 \pm 0.7 \mathrm{~kg}$ were randomly assigned into four treatments, with six replicates/group (fifteen pigs/replicate). Each group consisted to an equal number of gilts and barrows. All diets were composed of corn, wheat and soybean throughout the experiment. And diets for pigs during the finishing phase were equivalent to nutrient requirement, and the trace mineral requirement estimate was regarded as total trace mineral elements (TME). To test the effects of reduction of ME $(\mathrm{Cu}, \mathrm{Zn}, \mathrm{Fe}$ and $\mathrm{Mn})$ or supplementation with $\mathrm{CC}$, the ex- perimental treatments was designed in a $2 \times 2$ factorial arrangement (Table S1 in Supporting Information) and was as follows: (1) a corn-soybean meal diet (control, TME); (2) control diet with $1600 \mathrm{mg} \mathrm{kg}^{-1} \mathrm{CC}(\mathrm{TME}+\mathrm{CC})$; (3) reduced trace mineral elements (RME) diet (control, RME); and (4) reduced trace mineral elements diet with $1600 \mathrm{mg} \mathrm{kg}^{-1} \mathrm{CC}$ ( $\mathrm{RME}+\mathrm{CC})$. Coated cysteamine, containing $27 \%$ cysteamine hydrochloride, was supplied by Hangzhou King Techin Technology Co., Ltd (Hangzhou, China). The pigs were weighed at the beginning of the experiment (day 0 ) and the day of slaughter (day 21) to compute weight gains. At the end of the feeding experiment, one pig from each pen was randomly selected and slaughtered by exsanguination after electrical stunning $(250 \mathrm{~V}, 0.5 \mathrm{~A}$, for 5-6 s). Serum and the longissimus dorsi muscle samples were collected for analysis.

To assess the effect of coated cysteamine on meat quality in finishing pigs fed reduced trace minerals diet, we tested drip loss, $\mathrm{pH}$ and meat color of longissimus dorsi (Table S2 in Supporting Information). We found that dietary RME significantly increased the $\mathrm{pH}$ of the longissimus dorsi at 45 min post mortem $(P<0.05)$. It indicates that the $\mathrm{pH}$ of the longissimus dorsi at $45 \mathrm{~min}$ post mortem is increased and the $\mathrm{pH}$ decline from $45 \mathrm{~min}$ to $24 \mathrm{~h}$ shows an increasing trend in pigs fed a diet with the deletion of ME. The increasing $\mathrm{pH}$ decline from $45 \mathrm{~min}$ to $24 \mathrm{~h}$ post mortem could accelerate the development of rancidity in pork (Kim et al., 2016). However, previous research found that the $\mathrm{pH}$ at $45 \mathrm{~min}$ post mortem and $\mathrm{pH}$ decline were not affected by the trace mineral removal. The discrepancy can be attributed both to differing amounts of trace mineral addition and to environmental stress. The $\mathrm{pH}$ decline from $45 \mathrm{~min}$ to $24 \mathrm{~h}$ post mortem tended to increase in pigs fed the RME diet

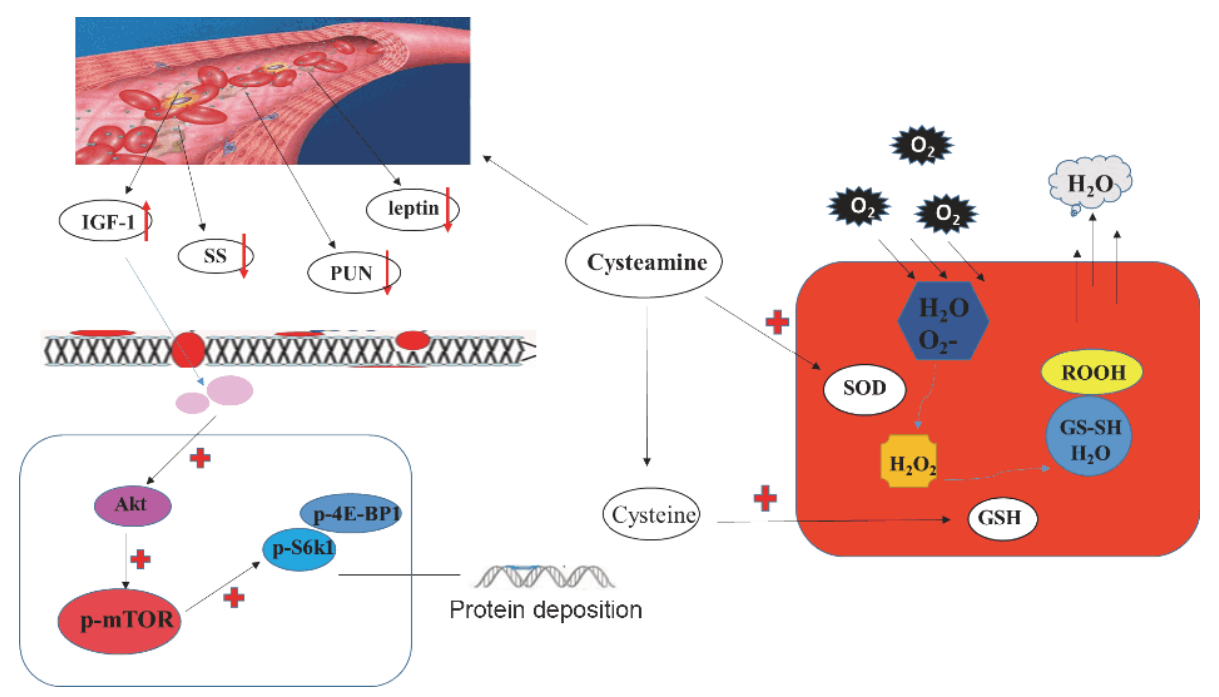

Figure 1 Effects of dietary cysteamine on improving antioxidant and accelerating protein deposition in the muscle of finishing pigs. (1) cysteamine could improve protein deposition by increasing the production of insulin-like growth factor (IGF-1) in the plasma and stimulating the Akt/mTOR signaling pathway; (2) cysteamine not only break the disulfide bond into cystine for synthesizing glutathione (GSH) but also increase the activity of superoxide dismutase (SOD) for improving antioxidant status. 
$(P<0.10)$. No significant difference related to either RME or $\mathrm{CC}$ was observed in other meat quality parameters. Based on the present results, the meat quality characteristics were not affected by the addition of CC or by RME diets. This was consistent with Wang et al. (2011), who indicated that cysteamine supplementation did not cause detrimental effects on meat quality. Nevertheless, Bai et al. (2018) reported that finishing pigs fed diets containing CC of $0.035 \mathrm{~g} \mathrm{~kg}^{-1}$ had a better meat colour at $24 \mathrm{~h}$ in the longissimus dorsi muscles. This difference might be related to different levels of supplementation.

We next investigated whether serum biochemical indices, amino acid composition and fatty acid composition of the longissimus dorsi muscles were affected when pigs fed supplemental coated cysteamine in reducing trace minerals diet. Amino acid content is determined to evaluate the biological value of muscle protein by following the procedure described by Lv et al. (2018). In the present study, addition of CC significantly enhanced $(P<0.05)$ the concentration of TAA, Cys, Cysthi and Orn, and had a tendency to increase $(P<0.10)$ the concentration of EAA and Gly (Table S3 in Supporting Information). This effect may enhance protein deposition through modifying amino acid metabolism in the pigs. As described by Zhou et al. (2015), cysteamine supplementation could promote protein deposition by stimulating the growth hormone (GH) secretion in skeletal muscle. The urea nitrogen balance indicates that adding $\mathrm{CC}$ to diets reduced the urea level in the longissimus dorsi muscle but increased the level in the serum. Moreover, reducing dietary TME tended to reduce $(P<0.10)$ the concentration of Leu and increase the concentrations of $\mathrm{Cit}(P<0.05)$ and $\operatorname{Tyr}(P<0.10)$ in the longissimus dorsi (Table S3 in Supporting Information). The levels of ME in diets have been shown to be closely related to protein metabolism. The results indicate that deletion of TME in diet has negative effects on protein retention in muscle. Besides, reducing dietary TME decreased $(P<0.05)$ the AST activity and LDH activity in the serum. And AST activity, as a biomarker for liver function and urea nitrogen and creatinine levels, is used to estimate glomerular filtration and renal functions. LDH, which catalyses the redox reaction between pyruvate and lactic acid, is an important enzyme in glycolysis and gluconeogenesis. The results indicate that the deletion of TME promotes the degradation of arginine and limits the process of amino acid synthesis, whereas supplementing an RME diet with CC enhances the concentrations of Cys and Cysthi. It seems likely that $\mathrm{CC}$ performs a compensatory action in finishing pigs fed an RME diet. However, further studies are required to investigate why the compensatory action of $\mathrm{CC}$ could be realized when reducing RME in diets.

Modification of the fatty acid composition of meat is strongly associated with differences in nutritional value and sensory attributes. Fatty acid composition of the longissimus dorsi was determined by direct transesterification described by Demirel et al. (2004). We found that no significant difference in the fatty acid composition of the longissimus dorsi was observed when TME in diets was reduced $(P>0.05)$. Dietary CC supplementation decreased $(P<0.05)$ the amount of c20:0 and c20:1 in the longissimus dorsi (Table S4 in Supporting Information). This might be associated with a reduction in somatostatin levels and an increase in GH secretion in the serum or tissue (Ma et al., 2016). Lipid mobilization is affected by the GH and other pituitary extracts. Thus, cysteamine may regulate fatty acid composition of the longissimus dorsi muscle by stimulating GH secretion. Furthermore, there are interaction effects on the quantity of c18:1n9t and PUFA in the longissimus dorsi. A higher PUFA content was associated with an improved meat flavour and accelerated pigment and lipid oxidation in meat (Ponnampalam et al., 2012).

We next investigated the effect of minerals content of the longissimus dorsi of pigs fed with an RME diet augmented by $\mathrm{CC}$. The contents of mineral substances (including $\mathrm{K}, \mathrm{Ca}$, $\mathrm{Cu}, \mathrm{Fe}, \mathrm{Mn}, \mathrm{Zn}, \mathrm{Cd}, \mathrm{Cr}$, and $\mathrm{Pb}$ ) was determined according to Cheng et al. (2016), through a Varian ICP-OES (ICP 720 ES; Agilent, USA). The results showed that dietary RME significantly decreased $(P<0.05)$ the concentration of $\mathrm{Cd}$ in the longissimus dorsi. The addition of $\mathrm{CC}$ to an RME diet had interaction effects on the concentration of $\mathrm{Cr}$ in the longissimus dorsi $(P<0.05)$ (Table S5 in Supporting Information). Meanwhile, our findings reveal that dietary RME decreases the content of $\mathrm{Ca}$ in the serum and reduces the deposition of $\mathrm{Ca}$ in muscle (Table S6 in Supporting Information). The level of serum calcium was closely related to the functions of muscle contraction and normal nerve conduction. Moreover, an RME diet reduced the deposition of $\mathrm{Cd}$, which poses a serious threat to human and animal health, in the longissimus dorsi. Therefore, the effect of RME on the deposition of beneficial trace elements in muscle was negative. Copper and $\mathrm{Mn}$ are involved in the synthesis of antioxidant enzymes, including cytosolic enzyme and superoxide dismutase, which cells from oxidative damage. Dietary CC significantly increased $(P<0.05)$ the concentration of $\mathrm{Cu}$, and had a tendency to increase $(P<0.10)$ the concentration of $\mathrm{Mn}$ in the longissimus dorsi (Table S5 in Supporting Information). The antioxidant capacity of cysteamine may be likely attributed to increasing the deposition of $\mathrm{Cu}$ and $\mathrm{Mn}$ in the muscle. The actual mechanism need to be further proven.

Understanding the scope of compensation effects of coated cysteamine on finishing pigs offered reduced trace minerals diet is critical for basic research and applications. Here we reveal that reduction of trace minerals in pig diets increased $\mathrm{pH}_{45 \text { min }}$ and decreased the concentration of $\mathrm{Cd}$ in the longissimus dorsi, and decreased serum $\mathrm{Ca}$ level, the activities of AST and LDH. It may cause a decrease in the deposition of minerals in the pork. But supplemented CC enhanced the 
contents of TAA, Cys, Cysthi and Orn, and increased the concentrations of $\mathrm{Cu}$ and $\mathrm{Mn}$, and reduced the levels of c20:0 and c20:1 in the longissimus dorsi. CC may have a compensatory effect on muscle nutrients deposition in finishing pigs fed a reduced trace minerals diet. We believe this contribution is theoretically and practically relevant because these findings can be applied in livestock industry.

Compliance and ethics The author(s) declare that they have no conflict of interests.

\begin{abstract}
Acknowledgements We thank Hangzhou King Techina Technology Company Academician Expert Workstation for providing technical assistance. This work was supported by the National Natural Science Foundation of China (31501964), the Youth Innovation Team Project of Institute of Subtropical Agriculture (ISA), China Academy Sciences (CAS) (2017QNCXTD_TBE), the China Agriculture Research System (CARS-35), the Natural Science Foundation of Guangdong Province (2017A030310410, 2017A030310398), and the National Natural Science Foundation of China (31790411).
\end{abstract}

\section{References}

Bai, M., Liu, H., Xu, K., Zou, B., Yu, R., Liu, Y., Xing, W., Du, H., Li, Y., and Yin, Y. (2018). Effects of dietary coated cysteamine hydrochloride on pork color in finishing pigs. J Sci Food Agric 98, 1743-1750.

Cheng, Y., Chen, Y., Li, X., Yang, W., Wen, C., and Zhou, Y. (2016). Effects of palygorskite inclusion on the growth performance, meat quality, antioxidant ability, and mineral element content of broilers. Biol Trace Elem Res 173, 194-201.

Demirel, G., Wachira, A.M., Sinclair, L.A., Wilkinson, R.G., Wood, J.D., and Enser, M. (2004). Effects of dietary $n-3$ polyunsaturated fatty acids, breed and dietary vitamin $\mathrm{E}$ on the fatty acids of lamb muscle, liver and adipose tissue. British J Nutrit 91, 551-565.

Kim, T.W., Kim, C.W., Yang, M.R., No, G.R., Kim, S.W., and Kim, I.S. (2016). Pork quality traits according to postmortem $\mathrm{pH}$ and temperature in Berkshire. Korean J Food Sci Animal Resour 36, 29-36.

Lönnerdal, B. (2000). Dietary factors influencing zinc absorption. J Nutrit 130, 1378S-1383S.

Li, S., Lu, L., Liao, X., Gao, T., Wang, F., Zhang, L., Xi, L., Liu, S., and Luo, X. (2016). Manganese elevates manganese superoxide dismutase protein level through protein kinase $\mathrm{C}$ and protein tyrosine kinase. Biometals 29, 265-274.

Lv, D., Xiong, X., Yang, H., Wang, M., He, Y., Liu, Y., and Yin, Y. (2018). Effect of dietary soy oil, glucose, and glutamine on growth performance, amino acid profile, blood profile, immunity, and antioxidant capacity in weaned piglets. Sci China Life Sci 61, 12331242.

Ma, W., Wu, J., Zhang, J., He, Y., Gui, J., and Mei, J. (2016). Sex differences in the expression of GH/IGF axis genes underlie sexual size dimorphism in the yellow catfish (Pelteobagrus fulvidraco). Sci China Life Sci 59, 431-433.

Nasr, M.A.F., Abd-Elhamid, T., and Hussein, M.A. (2017). Growth performance, carcass characteristics, meat quality and muscle aminoacid profile of different rabbits breeds and their crosses. Meat Sci 134, $150-157$.

Ponnampalam, E.N., Butler, K.L., McDonagh, M.B., Jacobs, J.L., and Hopkins, D.L. (2012). Relationship between muscle antioxidant status, forms of iron, polyunsaturated fatty acids and functionality (retail colour) of meat in lambs. Meat Sci 90, 297-303.

Wang, Y., Liu, G., Wang, Z., and Zhou, A. (2011). Effects of dietary supplementation with cysteamine on performance, carcass characteristics, meat quality and antioxidant status in finishing pigs. Agric Sci Technol 5, 14-19.

Zhou, P., Luo, Y., Zhang, L., Li, J., Zhang, B., Xing, S., Zhu, Y., Gao, F., and Zhou, G. (2017). Effects of cysteamine supplementation on the intestinal expression of amino acid and peptide transporters and intestinal health in finishing pigs. Anim Sci J 88, 314-321.

Zhou, P., Zhang, L., Li, J., Luo, Y., Zhang, B., Xing, S., Zhu, Y., Sun, H., Gao, F., and Zhou, G. (2015). Effects of dietary crude protein levels and cysteamine supplementation on protein synthetic and degradative signaling in skeletal muscle of finishing pigs. PLoS ONE 10, e0139393.

Zhu, D., Yu, B., Ju, C., Mei, S., and Chen, D. (2011). Effect of high dietary copper on the expression of hypothalamic appetite regulators in weanling pigs. J Anim Feed Sci 20, 60-70.

\section{SUPPORTING INFORMATION}

Table S1 Basal diet composition for finishing pigs

Table S2 Effects of coated cysteamine on meat quality in finishing pigs fed reduced trace minerals diet

Table S3 Effects of coated cysteamine on dorsal muscles free amino acid compositions in finishing pigs fed reduced trace minerals diet

Table S4 Effects of coated cysteamine on dorsal muscles fatty acid compositions in finishing pigs fed reduced trace minerals diet

Table S5 Effects of coated cysteamine on dorsal muscles mineral content in finishing pigs fed reduced trace minerals diet

Table S6 Effects of coated cysteamine on serum biochemical parameters in finishing pigs fed reduced trace minerals diet

The supporting information is available online at http://life.scichina.com and https://link.springer.com. The supporting materials are published as submitted, without typesetting or editing. The responsibility for scientific accuracy and content remains entirely with the authors. 